\title{
A simple game-theoretic model for upstream fish migration
}

\author{
Hidekazu Yoshioka ${ }^{1}$
}

Received: 28 December 2016/Accepted: 25 April 2017/Published online: 3 May 2017

(c) The Author(s) 2017. This article is an open access publication

\begin{abstract}
A simple game-theoretic model for upstream fish migration, which is a key element in life history of diadromous fishes, is proposed. Foundation of the model is a minimization problem on the cost of migration with the swimming speed and school size as the variables to be simultaneously optimized. Finding the optimizer ultimately reduces to solving a self-consistency equation without explicit solutions. Mathematical analytical results lead to the sufficient condition that the self-consistency equation has a unique solution, which turns out to be identified with the condition where the unique optimizer exists. Behavior of the optimizer is analyzed both mathematically and numerically to show its biophysical and ecological consequences. The analytical results demonstrate reasonable agreement between the present mathematical model and the theoretical and experimental results of upstream migration of fish schools reported in the past research.
\end{abstract}

Keywords Fish migration - Fish school - Game theory · Swimming speed $\cdot$ School size

\section{Introduction}

Migratory fishes, such as salmonids and sturgeons, are central aquatic species of environment and ecosystem of surface water systems, since they shape and create linkages among food webs as consumers, ecosystem engineers,

Hidekazu Yoshioka

yoshih@life.shimane-u.ac.jp

1 Faculty of Life and Environmental Science, Shimane University, Nishikawatsu-cho 1060, Matsue, Shimane 690-8504, Japan modulators of biochemical processes, and transport vectors (Winemiller and Jepsen 1998; Flecker et al. 2010). In addition, many of them are economically and culturally valuable fishery resources. Both their abundance and diversity have been highly affected by loss and degradation of habitats and migration routes (Guse et al. 2015; Logez et al. 2013; Radinger and Wolter 2015). Physical barriers, such as dams and weirs, are the major factors that fragment habitats and migration routes of fish (Jager et al. 2015; Yu and $\mathrm{Xu}$ 2016). Analyzing fish migration has, therefore, been a key topic in current biological and ecological research areas (Becker et al. 2015; Wang et al. 2012; White et al. 2011).

Since many of the fishes migrate by forming schools (Partridge and Pitcher 1980; Partridge 1982), analyzing migration dynamics of fish schools is a crucial topic. Migration of individuals and groups of organisms has been studied from both theoretical and practical viewpoints. For migration of isolated individual fishes, swimming path and velocity have been analyzed with the help of optimization theory where some hydrodynamic cost is minimized. The physiological energy minimization principle has been a core to determine the preferred swimming speed of individual fishes given a flow speed and water quality (Claireaux et al. 2006; Tucker 1975). Such models have recently been a basis of a generalized biological theory for the physiological energy minimization principle (Papadopoulos 2009, 2013). Some authors assumed that a migrating animal selects a path that minimizes the total resistance force (Bejan and Marden 2006; Lindberg et al. 2015; McElroy et al. 2012). Stöcker and Weihs (2001) analyzed a conceptual optimization model based on Weihs (1974) for energy-saving conceptual burst swimming of fishes. For migration of fish schools, increasing hydrodynamic efficiency for each individual has been considered as 
one of the most significant advantages to form schools (Mayer 2010). Increasing foraging efficiency and vigilance against predators have also been considered to be advantageous (Mayer 2010). On the other hand, increasing the foraging efficiency would not necessarily increase the net available food for each individual in a school (Beecham and Farnsworth 1999). Day et al. (2011) experimentally observed that smaller fish schools of Poecilia reticulate (guppy) demonstrated higher foraging efficiency than the larger ones.

Macroscopic models based on conceptualization for effectively analyzing animal group's dynamics have been proposed to comprehend group dynamics from phenomenological viewpoints. In these models, one of the most important parameters for describing migration of animal groups is the size: total number of individuals in the group. A common assumption shared by most of the models is that there is a size to compromise between advantages and disadvantages of forming groups. Gueron and Levin (1995) mathematically analyzed stability of the equilibrium of group size with the different fusion and fission rates. Anderson (1981) proposed a stochastic differential equation model for temporal evolution of the size of fish schools. Niwa (1996, 1998, 2005) established modelling frameworks for analyzing group-size distributions based on probabilistic descriptions of the merging and splitting phenomena.

On the other hand, microscopic, individual-based models with repulsion and attraction forces acting among the individuals have also been utilized to study group dynamics of animals. Behavior of individual-based deterministic and stochastic models for migrating fish schools has been studied both from mathematical and numerical viewpoints (Ebeling and Schweitzer 2001; Uchitane et al. 2015; Ngyuen et al. 2016; Ta et al. 2014). Sumpter et al. (2008) numerically investigated information transfer within moving animal groups using a 1-D time-discrete model of migration. They implied that each group has an optimal size where the group density is close to a phase transition point from disordered state to ordered state. Lee (2010) studied a noisy self-propelled particles system to analyze stability of collective motion of particles against period and intensity of the noise. Katz et al. (2011) evaluated repulsive and attractive forces acting among individuals of a fish school based on a trajectory tracking technique.

As reviewed above, both individual-based microscopic and conceptual macroscopic models have been proposed and extensively studied so far; however, the conventional theoretical research lacks analyzing migration of fish schools subject to water current, which are of great ecological and environmental interest. Important such examples include assessing passage efficiency of river reaches and hydraulic structures (Baek et al. 2015; Kerr et al. 2015; Vowles et al. 2015). There exist a series of extensive research results on behavior of fish schools subject to water current under experimental settings by Onitsuka and his coworkers, which can be effectively utilized for development and validation of mathematical models (Onitsuka et al. 2009, 2012a, b). Development of a conceptual model for the dynamics of fish schools based on these results can significantly advance comprehending and analyzing fish migration. This is the main motivation of this paper.

Based on the above research background, this paper presents a minimal mathematical model for upstream migration of fish schools in water current based on a simple game theory: an optimization theory. The model focuses on prolonged swimming behavior of fishes rather than burst ones, the latter being relevant for much shorter timescale. Game-theoretic models have extensively been utilized for ecological and biophysical modelling of animals (Kabalak et al. 2015; Mariani et al. 2016; Metz et al. 1992; Pereira and Martinez 2010; Riechert and Hammerstein 1983; Tanaka et al. 2011); however, application of such models to biophysics of fish migration is still rare. The variables to be optimized in the present model contain the swimming speed and school size that should be chosen by the fish school, so that the net cost of migration is minimized. The present model is an extended counterpart of the model for upstream migration of isolated individuals (Yoshioka and Shirai 2015; Yoshioka et al. 2015, 2016a). Assuming that certain simplifications reduce finding the optimizer to solving a self-consistency equation. Unfortunately, general exact expressions of its solution have not been found. To overcome this issue, this paper proposes an algorithm to numerically solve the self-consistency equation. An analytical estimate of the error between the true solutions and approximate solutions is also presented. Behavior of the optimizer is then mathematically and numerically studied to show its ecological and biophysical consequences. Consistency between the present model and the experimental results of swimming behavior of migratory fishes validates its performance.

The rest of this paper is organized as follows. Section 2 presents the mathematical model for upstream migration of isolated individuals. An extended mathematical model to deal with the migration of fish schools, which is the main focus of this paper, is also presented in this section. Section 3 performs mathematical and numerical analyses of the models. Section 4 concludes this paper and provides future perspectives of this research.

\section{Mathematical models}

This section presents two mathematical models for migration of isolated individual fishes and fish schools, the latter being an extension of the former. In this paper, an 
isolated fish is regarded as a fish school whose total number of individuals is exactly one. The models assume that the fishes are migrating toward upstream in a water current defined along the 1 -D space $\mathbb{R}$ with the flow velocity of $V>0$. A fish school is thus conceptualized as a particle moving along $\mathbb{R}$. The swimming velocity of the fish school is denoted as $u$, which gives its ground velocity as $V_{\mathrm{g}}=u-V$. The school is, therefore, migrating toward upstream when $V_{\mathrm{g}}>0$. The total number of individuals in the fish school is denoted as $N$, which is regarded as a continuous variable. The variable $N$ is hereafter referred to as the size, which is considered as another control variable to be optimized. Although the continuous approximation of the school size $N$, which is a discrete variable in reality, maybe problematic, it is well known that discrete optimization problems are far more technically and mathematically difficult than the continuous ones. Therefore, to avoid the above-mentioned difficulty, this paper focuses on the analysis with the school size $N$ as a continuous variable. This approximation becomes more accurate as $N$ increases (Niwa 2003). Although the conventional definition of the fish school (Partridge and Pitcher 1980; Partridge 1982) applies to $N \geq 3$, this paper assumes $N>0$ for the sake of simplicity of analysis. It should be noted that biologically relevant solutions should satisfy $N \geq 1$. The variable $u$ (the couple of variables $(u, N)$ ) is optimized in the model for isolated individual (fish school). We assume $u>V$, since the focus is upstream migration.

It should be noted that water current along a real open channel such as a river is not spatially homogenous. Such a situation is more realistic than that considered in this paper. We mention that the present model can serve as the basis of analyzing fish migration in the real open channels if it is effectively incorporated in that for the recently developed mathematical models based on the dynamic programming principle (Yoshioka 2016; Yoshioka et al. 2016b). This research topic is beyond the scope of this paper and will, therefore, be addressed elsewhere.

As shown in what follows, the present model has a number of key non-dimensional parameters, as summarized in Table 1.

\section{Model for upstream migration of an isolated individual}

\section{Ordinary differential equation}

The model for upstream migration of isolated individuals starts from the ODE

$\frac{\mathrm{d} X_{t}}{\mathrm{~d} t}=V-u$,

where $t$ is the time and $X_{t}$ is the 1-D position of the individual fish along the flow at the time $t$. The ODE (1) is equipped with the initial condition $X_{0}=0$, which is assumed without the loss of generality. The ODE (1) is then solved as

$X_{t}=(V-u) t$.

\section{Objective function}

The range $U$ of the variable $u$ is denoted as $U=[0,+\infty)$. Under this setting, it is possible to identify a swimming velocity as a swimming speed because of the non-negativity of the former. Assuming positive rheotaxis of the fish (Hinch et al. 2005; Keefer and Caudill 2014), the objective function $\phi(u)$ to be minimized through its migration process is formulated as

$\phi(u)=\int_{0}^{\tau} f(u) \mathrm{d} t$,

where $\tau$ is the time at which $X_{\tau}=L$, and thus, $\tau=L(u-V)^{-1}$. The objective function $\phi$ measures the total physiological energy consumed during the migration process. The cost function $f$ is assumed to be given by the power law-type function:

$f(u)=\frac{1}{n+1} u^{n+1}$,

with a parameter $n \geq 1$. The cost function $f$ in (4) shows that the fish is more high speed-averse for larger $n$, because it more rapidly grows for larger $n$. The cost function $f$ in (4) is convex and increasing with respect to $u$, which is in

Table 1 Summary of the key non-dimensional parameters

\begin{tabular}{lll}
\hline Parameter & Range or relationship & Meaning \\
\hline$n$ & $O\left(10^{0}\right)$ (Yoshioka et al. 2016a) & Growth rate of the hydrodynamic cost \\
$m$ & $O\left(10^{-1}\right)$ (Mayer 2010) & Discount rate of the hydrodynamic cost \\
$k$ & Larger than or equal to $O\left(10^{-1}\right)$ & Growth rate of the non-hydrodynamic cost \\
$y$ & $y=\frac{k(n+1)}{m+k}>1$ & Non-linearity of the self-consistency equation \\
$z$ & $z=\frac{m}{k n-m} \frac{1}{\tilde{z}}$, where $\tilde{z}=\left[\frac{m}{b k(n+1)}\left(\frac{y}{y-1} V\right)\right]^{\frac{k}{m+k}}$ & Inverse of the power function of the flow speed $V$ \\
\hline
\end{tabular}


good accordance with the conventional experimental results on swimming behavior of fishes (Brodersen et al. 2008; Cucco et al. 2012; Mori et al. 2015; Roche et al. 2013; Svendsen et al. 2010; Yoshioka et al. 2016a). The objective function is rewritten with (2) as

$\phi(u)=\frac{L}{n+1} \frac{u^{n+1}}{u-V}$.

Assuming that the migration process is temporally homogeneous, we can simply set $L=1$ without the loss of generality.

\section{Optimization problem}

According to the classical optimization theory (Bonnans et al. 2006), the optimal swimming speed $u^{*}$ that minimizes the objective function has to solve the equation derived from the first-order condition of optimality:

$f^{\prime}\left(u^{*}\right)\left(u^{*}-V\right)=f\left(u^{*}\right)$.

This Eq. (6) admits two solutions; one of them is $u^{*}=0$ and the other is

$u^{*}=\frac{n+1}{n} V>V$,

the latter being inferred as a biophysically relevant optimal swimming speed to represent upstream migration. Actually, it is straightforward to check that the candidate (7) minimizes $\phi$.

\section{Model for upstream migration of a fish school}

\section{Ordinary differential equation}

This section presents a mathematical model for upstream migration of a fish school. This paper assumes that longitudinal movement of the fish school is also described by the ODE (1) where the variable $u$ serves as the representative swimming velocity of the school.

\section{Objective function}

The value function for upstream migration of a fish school is different from that of an isolated individual due to the appearance of an additional control variable and coefficients. Assume that all the individuals in the school share an ideal objective function $\phi$, which is derived with a game-theoretic argument as explained below. The cost of swimming, namely, the hydrodynamic cost during the time interval $[0, \tau]$ is

$\phi_{1}(u, N)=\int_{0}^{\tau} \frac{f(u)}{\alpha(N)} \mathrm{d} t=\frac{L}{n+1} \frac{u^{n+1}}{(u-V) \alpha(N)}$, where the coefficient $\alpha \geq 1$ represents the discount of the hydrodynamic cost, which is motivated by the theoretically and experimentally validated fact that increasing the size $N$ can effectively reduce the hydrodynamic cost per individual (Marras et al. 2015; Mayer 2010). Using a conceptual model with probabilistic consideration, Mayer (2010) derived that the discount of the energy for each individual is proportional to $N^{-\frac{1}{3}}$, which leads to

$\alpha(N)=N^{m}$,

with $m=\frac{1}{3}$ in the present model.

The conventional research suggests that schooling has many aspects of benefits, such as improvements of navigational performance (Berdahl et al. 2014; Torney et al. 2015), hearing perception (Larsson 2009, 2012), and foraging efficiency (Di-Poi et al. 2014; Wang et al. 2016). These benefits are modelled in a lumped manner as

$\phi_{2}(u, N)=-\int_{0}^{\tau} a \mathrm{~d} t=\phi(u)=-\frac{L a}{u-V}$,

using a positive constant $a$. It seems to be reasonable to formulate an objective function $\phi$ by summing up $\phi_{1}$ and $\phi_{2}$ as $\phi=\phi_{1}+\phi_{2}$. However, this formulation leads to the trivial optimizer $N=+\infty$, which is not reasonable from a biological viewpoint. We, therefore, conjecture that this $\phi$ lacks the fact that schooling is not always definitely beneficial. Schooling would negatively affect collective performances of fishes, such as passage efficiency (Lemasson et al. 2014) and information transmission among individuals (Castellano et al. 2009; Shang and Bouffanais 2014). Other disadvantages of forming a school such as competitions among the individuals in the school would also exist. In addition, various environmental disturbances would also affect the fish migration. We thus assume that the net nonhydrodynamic cost of forming a school $\phi_{3}$ is formulated as

$$
\begin{aligned}
\phi_{3}(u, N, \eta) & =\int_{0}^{\tau}\left(\eta N^{q}-\frac{1}{c(p+1)} \eta^{p+1}\right) \mathrm{d} t \\
& =\frac{L}{u-V}\left(\eta N^{q}-\frac{1}{c(p+1)} \eta^{p+1}\right)
\end{aligned}
$$

where $p>0, q>0$, and $c>0$ are parameters, and $\eta \geq 0$ is the intensity of the negative effect of forming the school. The first term of (11) represents the cost of forming a school due to the above-mentioned negative influencing factors that increase as $N$ increases, while the second term represents the mitigation effect by forming a school.

The objective function $\phi$ for the fish school is consequently set as

$$
\begin{aligned}
\phi & =\phi_{1}+\phi_{2}+\phi_{3} \\
& =\frac{L}{u-V}\left(\frac{u^{n+1}}{(n+1) N^{m}}-a+\eta N^{q}-\frac{1}{c(p+1)} \eta^{p+1}\right) .
\end{aligned}
$$


The objective function is minimized by the fish school itself by choosing appropriate $(u, N)$, while nature maximizes it by choosing $\eta$. Since $L$ can be set as 1 without the loss of generality, the optimization problem reduces to the optimizer $(u, N, \eta)=\left(u^{*}, N^{*}, \eta^{*}\right)$ that gives

$\Phi=\inf _{u, N} \sup _{\eta} \phi(u, N, \eta)=\phi\left(u^{*}, N^{*}, \eta^{*}\right)$.

The optimization problem can be seen as a game with two non-cooperative players: the fish school and nature. Straightforward calculation shows

$\eta^{*}(N)=\eta^{*}=\left(c N^{q}\right)^{\frac{1}{p}}$

and

$$
\begin{aligned}
\Phi & =\sup _{u, N} \phi\left(u, N, \eta^{*}\right) \\
& =\sup _{u, N} \frac{1}{u-V}\left(\frac{u^{n+1}}{(n+1) N^{m}}-a+\frac{p}{p+1} c^{\frac{q}{p}} N^{\frac{q}{p}+1}\right) .
\end{aligned}
$$

Assuming mathematical consistency between the model of isolated individuals and that of fish schools, setting $N=$ 1 with the objective function $\phi$ in (12) should reduce to that in (5). This leads to the constraint of parameters

$a=\frac{p}{p+1} c^{\frac{q}{p}}=b$,

with a positive parameter $b$. Equation (15) is then rewritten with the positive parameter $k=1+\frac{q}{p}$ as

$$
\begin{aligned}
\Phi & =\sup _{u, N} \phi\left(u, N, \eta^{*}\right) \\
& =\sup _{u, N} \frac{1}{u-V}\left(\frac{u^{n+1}}{(n+1) N^{m}}+b\left(N^{k}-1\right)\right) .
\end{aligned}
$$

Relevant values of the parameters $n, m$, and $k$ can be to a certain extent inferred from theoretical and numerical analysis. On the parameter $n$, Yoshioka et al. (2016a) showed $O\left(10^{\circ}\right)$ for a variety of migratory fish species. Identification of the order of the parameter $k$ is more difficult than that for the parameters $n$ and $m$ because of its lumped nature. In this paper, it is assumed that the parameters $n, m$, and $k$ satisfy

$k n-m>0$,

which turns out to be a necessary condition for existence of an optimal $u^{*}$ with $u^{*}>V$. A biophysical interpretation of the condition (18) is that fishes create fish schools if they have to save the hydrodynamic cost of migration, which occurs if $k$ is large or $m$ is small. This is qualitatively consistent with the model of Stöcker and Weihs (2001). No biophysically relevant optimizer that represents upstream migration is obtained under this condition. The assumption shows that, provided that $m$ is around $O\left(10^{-1}\right)$ even if it is not $\frac{1}{3}$ (Mayer 2010), the order of the parameter $k$ can be inferred to be larger than or equal to $O\left(10^{-1}\right)$.

\section{Optimization problem}

By the static optimization problem, as in the case for the model of isolated individuals, the optimizers $u^{*}$ and $N^{*}$ have to solve the equations derived from the first-order necessary condition of optimality:

$\left(u^{*}\right)^{n}\left(u^{*}-V\right)=\frac{\left(u^{*}\right)^{n+1}}{(n+1)\left(N^{*}\right)^{m}}+b\left(\left(N^{*}\right)^{m+k}-1\right)$,

and

$\frac{m\left(u^{*}\right)^{n+1}}{(n+1)\left(N^{*}\right)^{m+1}}=b k\left(N^{*}\right)^{k-1}$.

With a technical calculation, the equations can be reduced to a self-consistency equation as follows. First, substituting (20) into (19) yields

$\left(u^{*}\right)^{n}\left(u^{*}-V\right)=\frac{1}{n+1}\left(u^{*}\right)^{n+1}\left[1+\frac{m}{k}\left\{1-\left(N^{*}\right)^{-k}\right\}\right]$.

Assuming that $u^{*}>0,(21)$ reduces to

$u^{*}=\frac{k(n+1)}{k n-m+m\left(N^{*}\right)^{-k}} V$.

Introduce the changes of variables

$$
\begin{gathered}
y=\frac{k(n+1)}{m+k}>1, \quad z=\frac{m}{k n-m} \frac{1}{\tilde{z}}, \quad \text { and } \\
\tilde{z}=\left[\frac{m}{b k(n+1)}\left(\frac{y}{y-1} V\right)\right]^{\frac{k}{m+k}} .
\end{gathered}
$$

The optimizers $u^{*}$ and $N^{*}$ are then expressed as

$u^{*}=\frac{y}{y-1} V w^{*} \quad$ and $\quad N^{*}=(\tilde{z})^{\frac{1}{k}}\left(w^{*}\right)^{\frac{y}{k}}$

respectively, with an auxiliary parameter $w^{*}$. The positivity of $u^{*}$ is guaranteed by (18), since $y>1$. The right side of (22) is further reduced to

$u^{*}=\frac{k(n+1)}{k n-m} V \frac{1}{1+\frac{m}{k n-m}\left(N^{*}\right)^{-k}}=\frac{y}{y-1} V \frac{\left(w^{*}\right)^{y}}{\left(w^{*}\right)^{y}+z}$.

By (25), the equation to be solved for $w^{*}$ is consequently found as

$w^{*}=F\left(w^{*}\right)=1-\frac{z}{\left(w^{*}\right)^{y}+z}$.

Finding the optimizer $\left(u^{*}, N^{*}\right)$ is thus ultimately reduced to solving the self-consistency equation (26). A relevant $w^{*}$ for upstream fish migration should give 
$u^{*}>V \quad$ and $\quad N^{*}>1$

Introduce the parameter

$\bar{z}=y^{-y}(y-1)^{y-1}$,

for the sake of brevity of descriptions. Straightforward calculation shows that the number of the intersections of the line $v=w$ and the curve $v=F(w)$ in the first quadrant of $v-w$ space is zero $(z>\bar{z})$, one $(\bar{z}=z)$, and two $(0<z<\bar{z})$. Figure 1 plots the graphs of $v=w$ and $v=$ $F(w)$ for $z>\bar{z}, \bar{z}=z$, and $0<z<\bar{z}$ in the square $[0,1] \times[0,1]$, which graphically validates the above-mentioned statement. For $\bar{z}=z$, in particular, $v=w$ is the tangent line of the curve $v=F(w)$ at the point $(\bar{w}, \bar{w})$, where $\bar{w}=\frac{y-1}{y}$. The above-mentioned geometrical information shows that there is no positive solution $w^{*}$ for large $z$, such that $z>\bar{z}$. In addition, for $\bar{z}=z, u^{*}$ is determined from (24) as $u^{*}=V$, which does not comply with (27). The analysis in what follows, therefore, assumes $0<z<\bar{z}$ where the two positive solutions $\bar{w}_{1}$ and $\bar{w}_{2}$, such that

$0<\bar{w}_{1}<\bar{w}<\bar{w}_{2}<1$,

solve (26) (Appendix). It turns out that the larger solution $w=\bar{w}_{2}$ is the biologically relevant solution to give $u^{*}>V$, which is denoted by $w^{*}$. The condition $0<z<\bar{z}$ can be expressed as $0<b<C V^{n+1}$ with a positive constant $C$

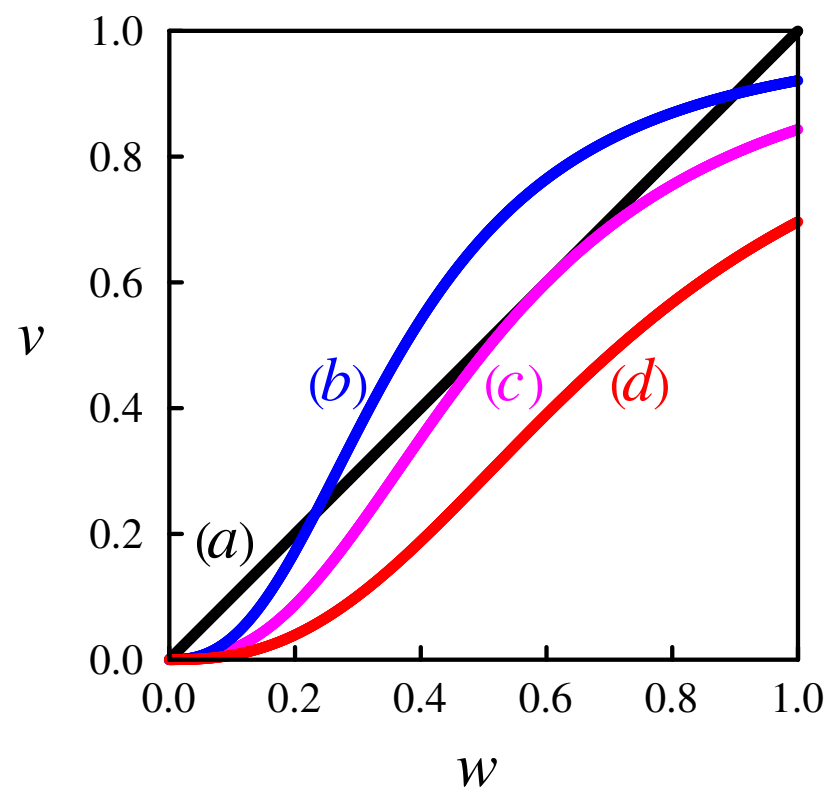

Fig. 1 Plots of the graphs of a the line $v=w$ and the curves $v=$ $F(w)$ for $\mathbf{b} z>\bar{z}, \mathbf{c} \bar{z}=z$, and $\mathbf{d} 0<z<\bar{z}$ in the unit square $[0,1] \times[0,1]$. This figure graphically shows the geometrical relationships between the line $v=w$ and the curve $v=F(w)$. They have zero, one (multiplicity one), and two positive solutions for $z>\bar{z}, \bar{z}=z$, and $0<z<\bar{z}$, respectively. For $\bar{z}=z$ in particular, $v=w$ is a tangent line of $v=F(w)$ at the point $(\bar{z}, \bar{z})$

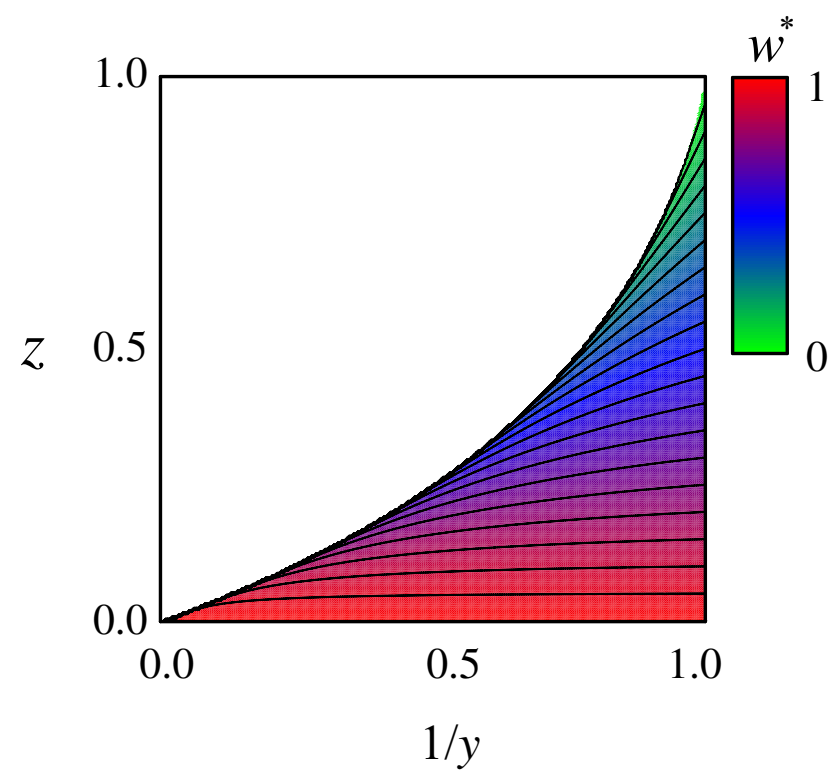

Fig. 2 2-D contour diagram of $w=w^{*}$ as a bi-variate function of $z$ and $y^{-1}$ for $0<z<\bar{z}$ and $0<y^{-1}<1$. The white region represents the area where $w^{*}$ does not exist. The curve that separates the white and colored regions in the figure corresponds to the curve $z$ in (32) (color figure online)

depending on neither $b$ nor $V$, meaning that the weight $b$ for the cost of schooling should not be too large to form a school.

Figure 2 shows a 2-D contour diagram of $w^{*}$ as a bivariate function of $z$ and $y^{-1}$ for $0<z<\bar{z}$ and $0<y^{-1}<1$. It should be noted that not every $w^{*}$ plotted in Fig. 2 complies with (27). This is because $k$ is expressed with (23) as $k=\frac{n+1-y}{m}>0$,

meaning that a relevant $y$ should satisfy

$\frac{1}{n+1}<y^{-1}<1$.

In Fig. 2, the boundary curve that separates the colored and white regions represents

$z(q)=q(1-q)^{\frac{1}{q}-1}\left(=y^{-y}(y-1)^{y-1}\right)$,

with $q=y^{-1}$, which is increasing and convex with respect to $q$.

\section{Analysis of the optimizers}

Qualitative and quantitative analyses of the optimizers $u^{*}$ and $N^{*}$ are performed to comprehend their dependence on the model parameters. The analysis is also intended to find appropriate ranges of the parameters to give realistic $u^{*}$ and $N^{*}$. 


\section{Qualitative analysis}

Qualitative analysis of the optimal controls $u^{*}$ and $N^{*}$ is first performed before the quantitative analysis in the next sub-section. The analysis here focuses on the signs of partial differentials of the optimal controls with respect to the model parameters, so that their biophysical and ecological consequences are clearly indicated.

Proposition 1 The solution $w=w^{*}$ to the self-consistency Eq. (26) is decreasing with respect to $z$ and $y$, namely, $\frac{\partial w^{*}}{\partial z}<0$ and $\frac{\partial w^{*}}{\partial y}<0$.

Proof of Proposition 1 Partially differentiating both sides of (26) with respect to $z$ leads to

$$
\begin{aligned}
\frac{\partial w^{*}}{\partial z} & =\frac{y\left(w^{*}\right)^{y-1} \frac{\partial w^{*}}{\partial z}}{\left(w^{*}\right)^{y}+z}-\left(w^{*}\right)^{y} \frac{y\left(w^{*}\right)^{y-1} \frac{\partial w^{*}}{\partial z}+1}{\left[\left(w^{*}\right)^{y}+z\right]^{2}} \\
& =F^{\prime}\left(w^{*}\right) \frac{\partial w^{*}}{\partial z}-\frac{1}{\left[\left(w^{*}\right)^{y}+z\right]^{2}},
\end{aligned}
$$

which is rewritten as

$$
\frac{\partial w^{*}}{\partial z}=-\frac{1}{\left[\left(w^{*}\right)^{y}+z\right]^{2}\left[1-F^{\prime}\left(w^{*}\right)\right]}<0,
$$

since $F^{\prime}(w)<0$ at $w=w^{*}$ by a geometrical consideration. Similarly, using the formula

$\frac{\partial}{\partial y} \ln \left[\left(w^{* y}\right)\right]=\ln \left(w^{*}\right)+y\left(w^{*}\right)^{-1} \frac{\partial w^{*}}{\partial y}$,

(26) leads to

$$
\begin{aligned}
\frac{\partial w^{*}}{\partial y} & =\frac{z}{\left[\left(w^{*}\right)^{y}+z\right]^{2}} \frac{\partial\left(w^{*}\right)^{y}}{\partial y} \\
& =\frac{z\left(w^{*}\right)^{y}}{\left[\left(w^{*}\right)^{y}+z\right]^{2}}\left[\ln \left(w^{*}\right)+y\left(w^{*}\right)^{-1} \frac{\partial w^{*}}{\partial y}\right] .
\end{aligned}
$$

Therefore, (36) shows

$$
\begin{aligned}
\frac{\partial w^{*}}{\partial y} & =\frac{z\left(w^{*}\right)^{y} \ln \left(w^{*}\right)}{\left[\left(w^{*}\right)^{y}+z\right]^{2}\left[1-F^{\prime}\left(w^{*}\right)\right]} \\
& <0,
\end{aligned}
$$

since $\ln \left(w^{*}\right)<0$.

Proposition 1 has biophysical meanings. By (24), the partial derivatives of $u^{*}$ and $N^{*}$ with respect to $V$ satisfy

$\frac{\partial u^{*}}{\partial V}>0 \quad$ and $\quad \frac{\partial N^{*}}{\partial V}>0$,

since $\frac{\partial z}{\partial V}<0$. In addition, the partial derivatives of $u^{*}$ and $N^{*}$ with respect to $b$ satisfy

$\frac{\partial u^{*}}{\partial b}<0$ and $\frac{\partial N^{*}}{\partial b}<0$, since $\frac{\partial z}{\partial b}<0$. The first inequality in (38) shows positive rheotaxis of the fish school, which has been reported to be satisfied in experimental fish migration for a series of $N$ against Plecoglossus altivelis (Ayu) (Onitsuka et al. 2012a). The second inequality in (38) shows that increasing the flow speed potentially increases the optimal number of individuals in a fish school, so that the physiological energy consumed by each individual is effectively reduced. This statement has also been experimentally validated against $P$. altivelis (Onitsuka et al. 2012a). The two inequalities in (39) show that the present mathematical model predicts that increasing the weight of the non-hydrodynamic forces a fish school to decide smaller size and slower swimming speed to be optimal.

Partial differentials of $y$ with respect to model parameters further highlight qualitative properties of the optimizers $u^{*}$ and $N^{*}$ associated with the present mathematical model. Rather than dealing with the parameters $m$ and $k$ separately, it is efficient to consider the hybrid parameter $m k^{-1}$ that quantifies the ratio of the discount rate of the energetic cost and the increasing rate of the schooling cost. The partial derivatives of $y$ with respect to $n$ and $m k^{-1}$ satisfy

$\frac{\partial y}{\partial n}>0$ and $\frac{\partial y}{\partial\left(m k^{-1}\right)}<0$,

respectively. The two inequalities in (40) lead to the inequalities

$\frac{\partial u^{*}}{\partial n}<0 \quad$ and $\quad \frac{\partial N^{*}}{\partial n}<0$.

Equation (40) then leads to

$\frac{\partial u^{*}}{\partial\left(m k^{-1}\right)}>0$ and $\quad \frac{\partial N^{*}}{\partial\left(m k^{-1}\right)}>0$.

The first inequality in (41) shows that a fish school with a more speed-averse energetic cost (the cost function with larger $n$ ) chooses a smaller swimming speed to be optimal, which associates a smaller school size as indicated in the second inequality. The first inequality in (42) indicates that a higher swimming speed is decided to be optimal for relatively higher efficiency of the physiological energy discount by forming a school. The second inequality in (42) shows that a larger school size is decided to be optimal as the decreasing rate of the energetic cost becomes higher than the increasing rate of the schooling cost.

Qualitative similarity between the model for isolated individuals and that for fish schools is also discussed in this paper. Straightforward calculation shows that the optimal control $u^{*}$ for the model of isolated individuals with the analytical formula (7) is decreasing with respect to $n$. By (41), the two models, therefore, predict $\frac{\partial u^{*}}{\partial n}<0$. In addition, 
by (7) and (38), they predict $\frac{\partial u^{*}}{\partial V}>0$. The latter statement for isolated individuals is in accordance with the experimental results (Onitsuka et al. 2012b). Actually, it is possible to derive a sharper estimate of the partial derivative $\frac{\partial u^{*}}{\partial V}$ as shown in the proposition below.

\section{Proposition 2 The model for fish school leads to}

$$
\frac{\partial u^{*}}{\partial V}>1 \text {. }
$$

Proof of Proposition 2 By (7) and (38), the inequality

$$
\frac{\partial w^{*}}{\partial V}=\frac{\partial}{\partial V}\left(\frac{u^{*}-V}{V}\right)=\frac{1}{V}\left[\frac{\partial u^{*}}{\partial V}-\frac{u^{*}}{V}\right]>0
$$

namely,

$\frac{\partial u^{*}}{\partial V}>\frac{u^{*}}{V}$

follows. Assuming that $u^{*}>V$ derives (43)

Proposition 2 shows that the optimal swimming speed $u^{*}$ increases as the flow speed $V$ does and the rate of its increase is larger than one. The inequality (43) theoretically leads to the statement that the difference $u^{*}-V$ is increasing with respect to $V$, which has been validated for upstream migration of fish schools in experimental flumes at least for not too large $V$ (Onitsuka et al. 2012b). The inequality also applies to the model for upstream migration of isolated individuals.

\section{Quantitative analysis}

Quantitative behavior of the optimizers $u^{*}$ and $N^{*}$ has been performed in the previous sub-section whose results are validated in this section through numerical analysis. Their behavior is also quantified here and realistic ranges of the model parameters are discussed.

To carry out the quantitative analysis, the value of the biologically relevant solution $w=w^{*}$ to Eq. (26) has to be accurately approximated; however, it has empirically been found that this is not possible for generic $y$. To overcome this difficulty, this paper applies a fixed-point iteration algorithm to generate an approximate sequence of the solution $w=w^{*}$. The algorithm is formulated as

$w_{r+1}=F\left(w_{r}\right)$ for $r=0,1,2, \ldots$,

subject to the initial guess $w_{0}=1$. The error between the approximate solution $w_{r}$ and the true solution $w^{*}$ is analytically estimated as

$0<w_{r}-w^{*}<\theta^{(y-1) r} \quad$ for $\quad r=0,1,2, \ldots$,

with $0<\theta=\frac{y z}{\bar{w}^{y-1}}<1$,

showing that this numerical algorithm can generate a decreasing sequence of approximated solutions that rigorously converges to the true solution. It is also shown that a similar algorithm with an initial guess $w_{0}=\bar{w}$ can also generate a convergent sequence with the limit $w^{*}$, which on the other hand is monotonically increasing. For each couple of the fixe parameter values of $y$ and $z$, the iteration algorithm is stopped at $r \geq 1$, such that $\left|w_{r+1}-w_{r}\right|<\varepsilon=10^{-10}$. Decreasing the value of the threshold $\varepsilon$, several orders of the magnitude do not affect the computational results presented below. Details of the iterative method for approximating solutions are described in Appendix.

The non-dimensional quantity $W^{*}=\frac{u^{*}-V}{V}$ is introduced here with biophysically more clear meaning (non-dimensionalized ground speed) than the quantity $w^{*}$ that has been employed for the sake of mathematical analysis. Figure 3a-f shows the contour diagrams of $W^{*}=\frac{u^{*}-V}{V}$ complying with the conditions (27) for the integers $1 \leq n \leq 6$. Figure $4 \mathrm{a}-\mathrm{f}$ shows the contour diagrams of $N^{*}$ complying with the conditions (27) for the integers $1 \leq n \leq 6$. The white region in each panel of Figs. 3 and 4 is the area where $W^{*}$ is smaller than 0 or $N^{*}$ is smaller than 1. Such optimal controls are not biologically relevant, since at least $N^{*}$ should be larger than or equal to 1 and an upstream migration should correspond to a positive $W^{*}$. The computational results presented in Figs. 3 and 4 are consistent with the mathematical analytical results presented in the previous sub-section: $W^{*}$ and $N^{*}$ are decreasing with respect to $z$ in (23), namely, increasing with respect to $V$.

The flow speed $V$ in a river under calm conditions is typically from $O\left(10^{-1}\right) \mathrm{m} / \mathrm{s}$ to $O\left(10^{0}\right) \mathrm{m} / \mathrm{s}$, and would be at most $O\left(10^{1}\right) \mathrm{m} / \mathrm{s}$. In addition, the maximum sustained swimming speed of fish living in rivers is at most $O\left(10^{0}\right) \mathrm{m} / \mathrm{s}$ (Onitsuka et al. 2009). Therefore, the biologically relevant order of the non-dimensional quantity $W^{*}$ can be at most $O\left(10^{1}\right)$, which roughly corresponds to green-to-blue area in each panel of Fig. 3. It would not be unrealistic to assume that the range of $N^{*}$ includes the range $1<N^{*}<100$. Figure $5 \mathrm{a}-\mathrm{f}$ shows the area with $0<W^{*}<100$ and $1<N^{*}<100$ for the integers $1 \leq n \leq 6$. These figures show that increasing $n$ yields wider area satisfying the above-mentioned ranges of $0<W^{*}<100$ and $1<N^{*}<100$. Figure 5 indicates that the lower right end of the area complying with these conditions is more insensitive to the lower left-end, which has been checked to be true for $n$ up to 10 . 

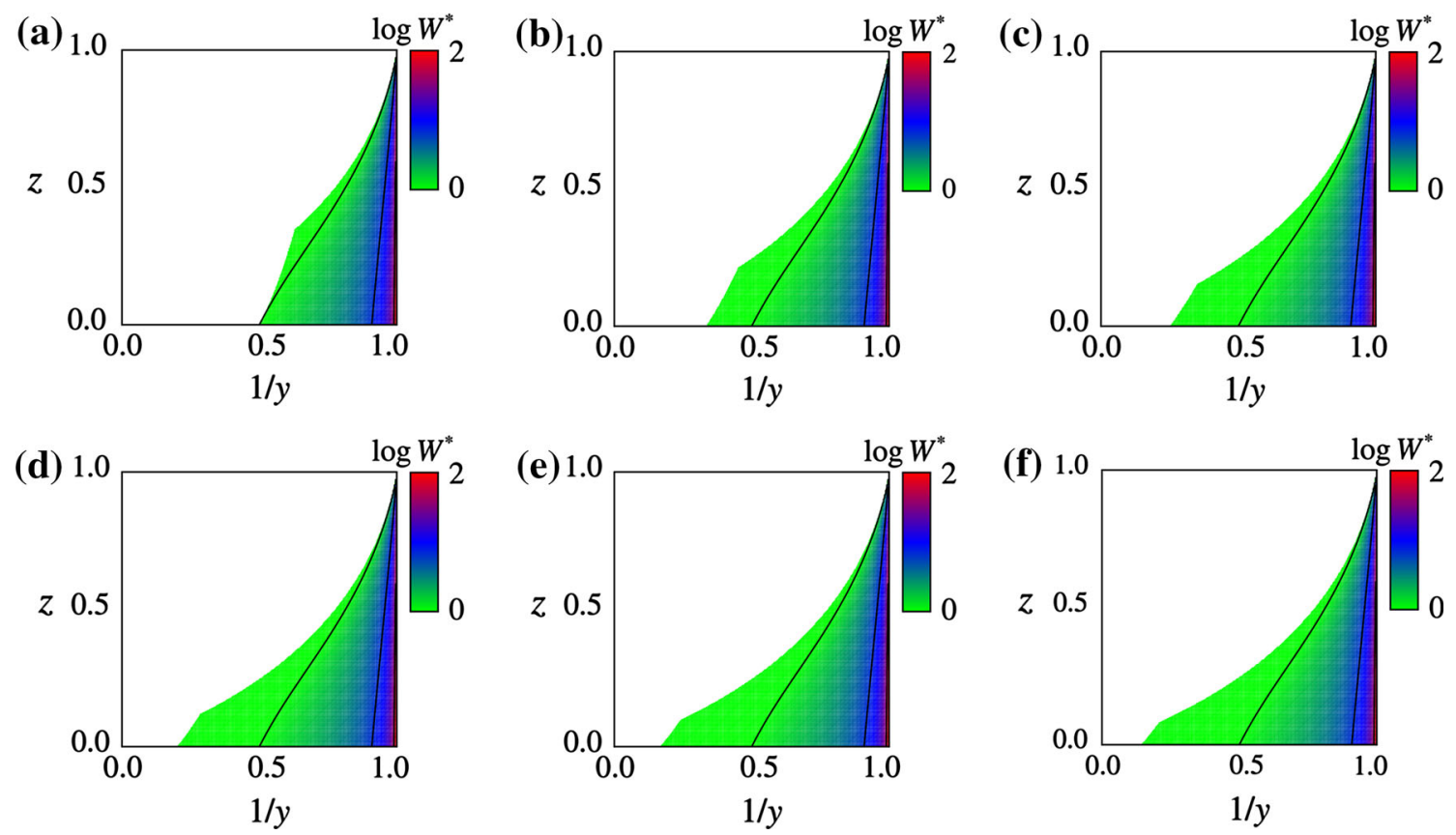

Fig. 3 Contour diagrams of $W^{*}=\frac{u^{*}-V}{V}$ for $0<z<\bar{z}$ and $0<y^{-1}<1$ with a $n=1$, b $n=2$, c $n=3$, d $n=4$, e $n=5$, and $\mathbf{f} n=6$. The white region represents the area where $w^{*}$ does not exist as in Fig. 2 or $W^{*}<0$, which does not verify necessary conditions for relevant solutions
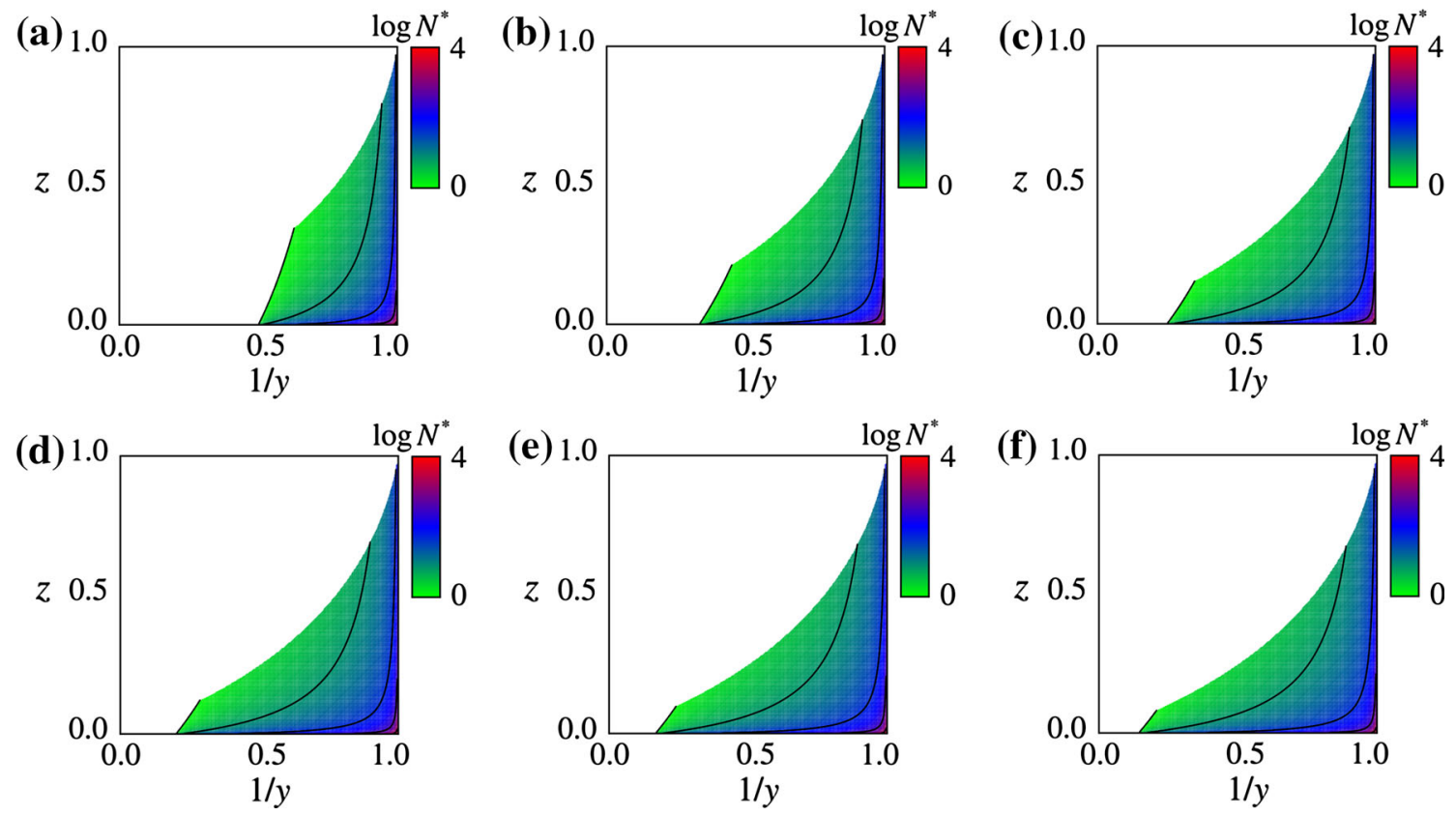

Fig. 4 Contour diagrams of $N^{*}$ for $0<z<\bar{z}$ and $0<y^{-1}<1$ with a $n=1$, $\mathbf{b} n=2$, $\mathbf{c} n=3$, $\mathbf{d} n=4$, e $n=5$, and $\mathbf{f} n=6$. The white region represents the area where $w^{*}$ does not exist as in Fig. 2 or $N^{*}<1$, which does not verify necessary conditions for relevant solutions

\section{Conclusions}

A minimal mathematical model for upstream migration of fish schools in 1-D environment based on a game-theoretic formulation was presented and behavior of the associated optimizers was analyzed both mathematically and numerically. The optimization problem was effectively reduced to solving a self-consistency equation that was not explicitly solved, but its qualitative solution behavior could be analyzed with a course of elementary calculation. Dependence of the optimal swimming speed $u^{*}$ and the optimal size $N^{*}$ of fish schools on the model parameters was theoretically analyzed for comprehending their qualitative behavior. The analytical results obtained in 

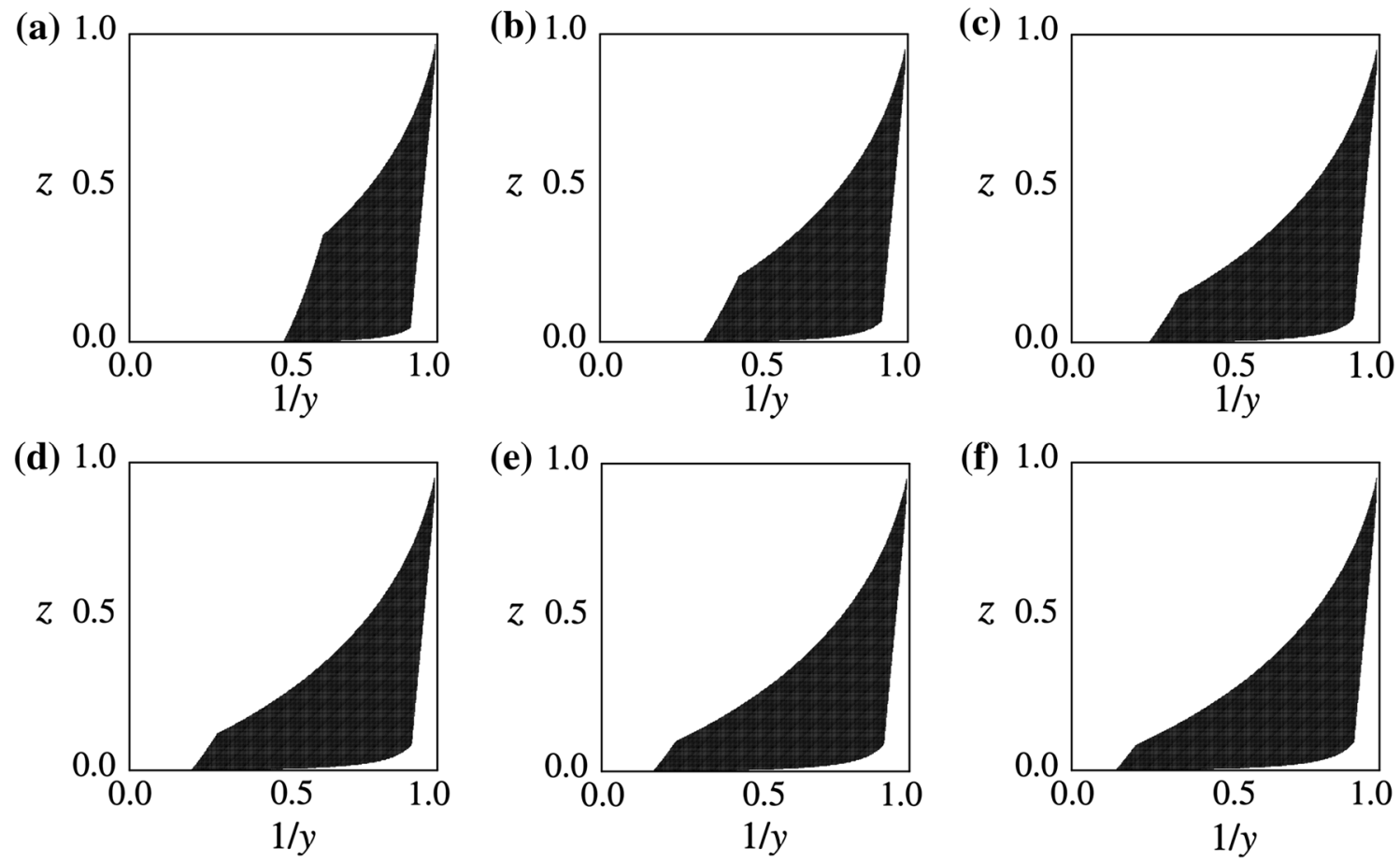

Fig. 5 Diagrams of the area complying with the conditions $0<W^{*}<100$ and $1<N^{*}<100$ with a $n=1$, b $n=2$, c $n=3$, d $n=4$, e $n=5$, and $\mathbf{f} n=6$, which are considered to be biologically

relevant in this paper. The white region represents the area where these conditions are not satisfied

"Qualitative analysis" showed that the dependence of the optimal swimming speed $u^{*}$ and the school size $N^{*}$ derived from the present mathematical model on the flow speed $V$ qualitatively agrees well with the experimental results (Onitsuka et al. 2012a, b). This paper thus provided a theoretical explanation of the experimental results from a game-theoretic viewpoint, which is a first attempt to the author's knowledge. Numerical analysis with the help of a mathematically rigorous technique of fixed-point iteration generated a monotonically increasing sequence of approximate solutions to the self-consistency equation. The computed solutions to the self-consistency equation with the appropriate ranges of the model parameters revealed quantitative behavior of the optimal controls, which validated the theoretical results obtained from the qualitative analysis. The ranges of the realistic model parameter values were estimated from the computational results. Currently, we are planning to perform artificially hatching experiments of $P$. altivelis in a river with local fishery cooperatives for the identification of the model parameters involved in the objective function.

This paper could quantify dependence of the optimal controls $u^{*}$ and $N^{*}$ on the model parameters under simplified conditions, so that the most part of the analysis can be performed exactly. Theoretically, it is possible to choose more complex parameterization of the coefficients to more appropriately model migration dynamics of fish schools

when necessary. However, analyzing such an extended model would require the use of a sophisticated numerical technique and considering an unnecessarily complicated model would not be beneficial from both theoretical and practical viewpoints. The present mathematical model can be a minimal model for migration of fish schools where the flow of water and both advantages and disadvantages are taken into account. Its practical extension includes considering unsteady and inhomogeneous flow field in the ODE for longitudinal movements of fish schools. The analytical results presented in this paper can be to some extent valid for analyzing behavior of the optimal controls when the rate of change of the flow speed $V$ is not very large, so that it does not exceeds the maximum swimming speed of the individual fishes. Future research will use the present mathematical models for analyzing upstream fish migration in existing river systems in Japan as their real applications with the help of numerical simulation techniques. Applicability of the present mathematical modelling framework to migration of other animals, such as migratory birds (McLaren et al. 2014; Vansteelant et al. 2017), will also be examined in future.

Acknowledgements The River Fund No. 285311020 in charges of The River Foundation, JSPS Research Grant No. 15H06417 and No. 17K15345, and WEC Applied Ecology Research Grant No. 2016-02 support this research. The author thanks to the reviewer for providing valuable comments and suggestions. 
Open Access This article is distributed under the terms of the Creative Commons Attribution 4.0 International License (http://crea tivecommons.org/licenses/by/4.0/), which permits unrestricted use, distribution, and reproduction in any medium, provided you give appropriate credit to the original author(s) and the source, provide a link to the Creative Commons license, and indicate if changes were made.

\section{Appendix: Numerical algorithms}

This supplementary material presents monotone iteration algorithms for approximating the largest positive solution to the self-consistency equation

$w=F(w)=\frac{w^{y}}{w^{y}+z}$,

with the parameters $y$ and $z$, such that $y>1$ and $0<z<\frac{(y-1)^{y-1}}{y^{y}}$. Under the stated conditions, the Eq. (49) admits two positive solutions $\bar{w}_{1}$ and $\bar{w}_{2}$, such that

$0<\bar{w}_{1}<\bar{w}<\bar{w}_{2} \equiv w^{*}<1$,

with $\bar{w}=\frac{y-1}{y}$. Straightforward calculation shows

$\bar{w}-F(\bar{w})=\frac{1}{\bar{w}^{y}+z} \frac{y-1}{y}\left(\frac{(y-1)^{y-1}}{y^{y}}-z\right)>0$,

$F^{\prime}(w)=\frac{y z w^{y-1}}{\left(w^{y}+z\right)^{2}}>0$ for $w>0$,

and

$F^{\prime \prime}(w)=\frac{y(y+1) z w^{y-2}}{\left(w^{y}+z\right)^{3}}\left(\frac{y-1}{y+1}-w^{y}\right)$ for $\quad w>\bar{w}$.

In addition, it is also shown that

$w<F(w)$ for $\bar{w}_{1}<w<\bar{w}_{2}$.

Combining (49) and (52) yields the estimate

$0<F^{\prime}\left(w^{*}\right)=\frac{y z}{\left(w^{*}\right)^{y+1}} \frac{\left(w^{*}\right)^{y}}{\left(w^{*}\right)^{y}+z} \frac{\left(w^{*}\right)^{y}}{\left(w^{*}\right)^{y}+z}=\frac{y z}{\left(w^{*}\right)^{y-1}}$,

which reduces to

$$
\begin{aligned}
0 & <F^{\prime}\left(w^{*}\right) \\
& <\frac{y z}{\bar{w}^{y-1}} \\
& <\frac{y}{\bar{w}^{y-1}} \frac{(y-1)^{y-1}}{y^{y}} \\
& =1 .
\end{aligned}
$$

Therefore, (56) shows

$0<F^{\prime}\left(w^{*}\right)<\frac{y z}{\bar{w}^{y-1}} \equiv \theta<1$.
Combining (53) and (55) then yields

$0<F^{\prime}(w)<\theta$ for $w^{*}>w$.

The present algorithm is thus a fixed-point type with a contraction property whose convergence is guaranteed under the conditions assumed in this paper (Atkinson and Han 2005, Chapter 5).

Two iteration algorithms for finding the solution $w=w^{*}$, which is a biophysically relevant solution, are presented in what follows. The first (second) algorithm generates a monotonically decreasing (increasing) sequence of approximated solutions.

The first algorithm is presented as

(First algorithm)

$w_{0}=1 \quad$ and $\quad w_{r+1}=F\left(w_{r}\right)$ for $r \geq 0$.

On the other hand, the second algorithm is presented as

(Second algorithm)

$w_{0}=\bar{w} \quad$ and $\quad w_{r+1}=F\left(w_{r}\right)$ for $\quad r \geq 0$.

The only apparent difference between the two algorithms is the initial guess $w_{0}$. The following theorems hold for these algorithms.

Theorem 1 The sequence $w_{r}$ generated by the first algorithm satisfies

$w^{*}<\cdots<w_{r}<\cdots<w_{1}<w_{0}=1$,

and converges to $w^{*}$.

Theorem 2 The sequence $w_{r}$ generated by the second algorithm satisfies

$0<\bar{w}=w_{0}<w_{1}<\cdots<w_{r}<\cdots<w^{*}$,

and converges to $w^{*}$.

Proof of Theorem 1 By (51), the inequality

$$
\begin{aligned}
w_{1}-w_{0} & =F\left(w_{0}\right)-1 \\
& =F(1)-1 \\
& <0
\end{aligned}
$$

holds. The inequality

$w_{0}-w^{*}=1-w^{*}>0$

is obvious. Assume

$w^{*}<w_{r}<\cdots<w_{1}<w_{0}=1$,

for a natural number $l=r$. Then, for $l=r+1$, the inequality

$$
\begin{aligned}
w_{r+1}-w_{r} & =F\left(w_{r}\right)-w_{r} \\
& =F\left(w_{r}\right)-F\left(w_{r-1}\right) \\
& <0
\end{aligned}
$$

follows from (52) and (65). In addition, the inequality 


$$
\begin{aligned}
w^{*}-w_{r+1} & =F\left(w^{*}\right)-w_{r+1} \\
& =F\left(w^{*}\right)-F\left(w_{r}\right) \\
& <0
\end{aligned}
$$

also follows from (52) and (65). Convergence of the sequence $w_{r}$ follows from its monotonicity and boundedness. The limit of the sequence is unique and is clearly $w^{*}$. Proof of Theorem 1 completes by the application of an inductive argument. Theorem 2 can be proven in an essentially similar way.

Remark 3 It is possible to analytically derive a theoretical convergence estimate of the first algorithm. By (58), the sequence $\left|w_{r}-w^{*}\right|=c_{r}$ satisfies

$$
\begin{aligned}
c_{r+1} & =\left|w_{r+1}-w^{*}\right| \\
& =\left|F\left(w_{r}\right)-F\left(w^{*}\right)\right| \text { for } \quad r \geq 0 \\
& \leq \theta^{y-1}\left|w_{r}-w^{*}\right| \\
& =\theta^{y-1} c_{r},
\end{aligned}
$$

which leads to

$c_{r} \leq \theta^{(y-1) r} c_{0}<\theta^{(y-1) r} \quad$ for $\quad r \geq 0$,

since $c_{0}=\left|1-w^{*}\right|<1$. The convergence estimate which does not explicitly depend on is derived as follows:

$c_{r}<\theta^{(y-1) r} \quad$ for $\quad r \geq 0$.

\section{References}

Anderson JJ (1981) A stochastic model for the size of fish schools. Fish B-NOAA 79:315-323

Atkinson K, Han W (2005) Theoretical numerical analysis. Springer, Berlin

Baek KO, Ku YH, Kim DY (2015) Attraction efficiency in naturallike fishways according to weir operation and bed change in Nakdong River, Korea. Ecol Eng 84:569-578

Becker A, Holland M, Smith JA, Suthers IM (2015) Fish movement through an estuary mouth is related to tidal flow. Estuar Coasts 39:1199-1207

Beecham JA, Farnsworth KD (1999) Animal group forces resulting from predator avoidance and competition minimization. $\mathrm{J}$ Theor Biol 198:533-548

Bejan A, Marden JH (2006) Unifying constructal theory for scale effects in running, swimming and flying. J Exp Biol 209:238-248

Berdahl A, Westley PA, Levin SA, Couzin ID, Quinn TP (2014) A collective navigation hypothesis for homeward migration in anadromous salmonids. Fish Fish 17:525-542

Bonnans JF, Gilbert JC, Lemaréchal C, Sagastizábal CA (2006) Numerical optimization: theoretical and practical aspects. Springer Science \& Business Media, Berlin

Brodersen J, Nilsson PA, Ammitzbøl J, Hansson LA, Skov C, Brönmark C (2008) Optimal swimming speed in head currents and effects on distance movement of winter-migrating fish. PLoS One 3:1-7
Castellano C, Fortunato S, Loreto V (2009) Statistical physics of social dynamics. Rev Modern Phys 81:591-646

Claireaux G, Couturier C, Groison AL (2006) Effect of temperature on maximum swimming speed and cost of transport in juvenile European sea bass (Dicentrarchus labrax). J Exp Biol 209:3420-3428

Cucco A et al (2012) A metabolic scope based model of fish response to environmental changes. Ecol Model 237:132-141

Day RL et al (2011) Interactions between shoal size and conformity in guppy social foraging. Anim Behav 62:917-925

Di-Poi C, Lacasse J, Rogers SM, Aubin-Horth N (2014) Extensive behavioural divergence following colonisation of the freshwater environment in three spine sticklebacks. PLoS One 9:e98980

Ebeling W, Schweitzer F (2001) Swarms of particle agents with harmonic interactions. Theory Biosci 120:201-224

Flecker AS, McIntyre PB, Moore JW, Anderson JT, Taylor BW, Hall RO Jr (2010) Migratory fishes as material and process subsidies in riverine ecosystems. In community ecology of stream fishes: concepts, approaches, and techniques. Am Fish Soc Symp 73:559-592

Gueron S, Levin SA (1995) The dynamics of group formation. Math Biosci 128:243-264

Guse B et al (2015) Eco-hydrologic model cascades: simulating land use and climate change impacts on hydrology, hydraulics and habitats for fish and macroinvertebrates. Sci Total Environ 533:542-556

Hinch SG, Cooke SJ, Healey MC, Farrell AT (2005) Behavioural physiology of fish migrations: salmon as a model approach. Fish Physiol 24:239-295

Jager HI, Efroymson RA, Opperman JJ, Kelly MR (2015) Spatial design principles for sustainable hydropower development in river basins. Renew Sustain Energy Rev 45:808-816

Kabalak A, Smirnova E, Jost J (2015) Non-cooperative game theory in biology and cooperative reasoning in humans. Theory Biosci 134:17-46

Katz Y et al (2011) Inferring the structure and dynamics of interactions in schooling fish. PNAS 108:18720-18725

Keefer ML, Caudill CC (2014) Homing and straying by anadromous salmonids: a review of mechanisms and rates. Rev Fish Biol Fish 24:333-368

Kerr JR, Karageorgopoulos P, Kemp PS (2015) Efficacy of a sidemounted vertically oriented bristle pass for improving upstream passage of European eel (Anguilla anguilla) and river lamprey (Lampetra fluviatilis) at an experimental Crump weir. Ecol Eng 85:121-131

Larsson M (2009) Possible functions of the octavolateralis system in fish schooling. Fish Fish 10:344-353

Larsson M (2012) Incidental sounds of locomotion in animal cognition. Anim Cogn 15:1-13

Lee CF (2010) Fluctuation-induced collective motion: a singleparticle density analysis. Phys Rev E 81:031125

Lemasson BH, Haefner JW, Bowen MD (2014) Schooling increases risk exposure for fish navigating past artificial barriers. PLoS One 9:e108220

Lindberg DE, Leonardsson K, Lundqvist H (2015) Path selection of Atlantic Salmon (Salmo salar) migrating through a fishway. River Res Appl 32:795-803

Logez M, Bady P, Melcher A, Pont D (2013) A continental-scale analysis of fish assemblage functional structure in European rivers. Ecography 36:80-91

Mariani P, Křivan V, MacKenzie BR, Mullon C (2016) The migration game in habitat network: the case of tuna. Theor Ecol 9:219-232

Marras S et al (2015) Fish swimming in schools save energy regardless of their spatial position. Behav Ecol Sociobiol 69:219-226 
Mayer P (2010) Economic models of fish shoal (school) size: a near comprehensive view of single species shoaling strategy. J Bioecon 12:119-143

McElroy B, DeLonay A, Jacobson R (2012) Optimum swimming pathways of fish spawning migrations in rivers. Ecology 1:29-34

McLaren JD, Shamoun-Baranes J, Dokter AM, Klaassen RH, Bouten W (2014) Optimal orientation in flows: providing a benchmark for animal movement strategies. J R Soc Interface 11:20140588

Metz JA, Nisbet RM, Geritz SA (1992) How should we define 'fitness' for general ecological scenarios? Trends Ecol Evol 7:198-202

Mori T, Miyata N, Aoyama J, Niizuma Y, Sato K (2015) Estimation of metabolic rate from activity measured by recorders deployed on Japanese sea bass Lateolabrax japonicus. Fish Sci 81:871-882

Ngyuen NLT, Ta VT, Yagi A (2016) Obstacle avoiding patterns and cohesiveness of fish school. J Theor Biol 406:116-123

Niwa HS (1996) Mathematical model for the size distribution of fish schools. Comput Math Appl 32:79-88

Niwa HS (1998) Migration dynamics of fish schools in heterothermal environments. J Theor Biol 193:215-231

Niwa HS (2003) Power-law versus exponential distributions of animal group sizes. J Theor Biol 224:451-457

Niwa HS (2005) Power-law scaling in dimension-to-biomass relationship of fish schools. J Theor Biol 235:419-430

Onitsuka K, Akiyama J, Yamamoto A, Watanabe T, Waki T (2009) Study on burst speed of several fishes living in rivers. J JSCE Ser B 65:296-307 (In Japanese with English Abstract)

Onitsuka K, Akiyama J, Mihara K, Shiraoka B (2012a) Effects of velocity on swimming behavior of a quintet of Ayu. J JSCE Ser B 68:239-244 (In Japanese with English Abstract)

Onitsuka K et al (2012b) Influence of sidewall on swimming behavior of isolated Ayu, Plecoglossus Altivelis Altivelis. J JSCE Ser B1 68:661-666 (In Japanese with English Abstract)

Papadopoulos A (2009) Hydrodynamics-based functional forms of activity metabolism: a case for the power-law polynomial function in animal swimming energetics. PLoS One 4:e4852

Papadopoulos A (2013) Relating models of activity metabolism to the metabolic efficiency of steady swimming. Open J Anim Sci 3:314-320

Partridge BL (1982) The structure and function of fish schools. Sci Am 246:90-99

Partridge BL, Pitcher TJ (1980) The sensory basis of fish schools. J Comput Physiol 135:315-325

Pereira MA, Martinez AS (2010) Pavlovian prisoner's dilemmaanalytical results, the quasi-regular phase and spatio-temporal patterns. J Theor Biol 265:346-358

Radinger J, Wolter C (2015) Disentangling the effects of habitat suitability, dispersal and fragmentation on the distribution of river fishes. Ecol Appl 25:914-927

Riechert SE, Hammerstein P (1983) Game theory in the ecological context. Annu Rev Ecol Syst 14:377-409

Roche DG, Binning SA, Bosiger Y, Johansen JL, Rummer JL (2013) Finding the best estimates of metabolic rates in a coral reef fish. J Exp Biol 216:2103-2110

Shang Y, Bouffanais R (2014) Influence of the number of topologically interacting neighbors on swarm. Sci Rep 4:4184

Stöcker S, Weihs D (2001) Optimization of energetic advantages of burst swimming of fish. Math Methods Appl Sci 24:1387-1400

Sumpter D, Buhl J, Biro D, Couzin I (2008) Information transfer in moving animal groups. Theory Biosci 127:177-186

Svendsen JC, Tudorache C, Jordan AD, Steffensen JF, Aarestrup K, Domenici P (2010) Partition of aerobic and anaerobic swimming costs related to gait transitions in a labriform swimmer. J Exp Biol 213:2177-2183

Ta VT, Linh NTH, Yagi A (2014) Flocking and non-flocking behavior in a stochastic Cucker-Smale system. Anal Appl 12:63-73

Tanaka Y, Iguchi K, Yoshimura J, Nakagiri N, Tainaka K (2011) Historical effect in the territoriality of ayu fish. J Theor Biol 268:98-104

Torney CJ, Lorenzi T, Couzin ID, Levin SA (2015) Social information use and the evolution of unresponsiveness in collective systems. J R Soc Interface 12:20140893

Tucker VA (1975) The energetic cost of moving about. Am Sci 63:413-419

Uchitane T, Ta VT, Yagi A (2015) An ordinary differential equation model for fish schooling. Scientiae Mathematicae Japonicae Online e-2012:415-426

Vansteelant WM, Shamoun-Baranes J, McLaren J, van Diermen J, Bouten W (2017) Soaring across continents: decision-making of a soaring migrant under changing atmospheric conditions along an entire flyway. J Avian Biol. doi:10.1111/jav.01298 (in press)

Vowles AS, Don AM, Karageorgopoulos P, Worthington TA, Kemp PS (2015) Efficiency of a dual density studded fish pass designed to mitigate for impeded upstream passage of juvenile European eels (Anguilla anguilla) at a model Crump weir. Fish Manag Ecol 22:307-316

Wang CY et al (2012) Migrations and movements of adult Chinese sturgeon Acipenser sinensis in the Yangtze River, China. J Fish Biol 81(2):696-713

Wang X, Pan Q, Kang Y, He M (2016) Predator group size distributions in predator-prey systems. Ecol Complex 26:117-127

Weihs D (1974) Energetic advantages of burst swimming of fish. J Theor Biol 48:215-229

White LJ, Harris JH, Keller RJ (2011) Movement of three nonsalmonid fish species through a low-gradient vertical-slot fishway. River Res Appl 27:499-510

Winemiller KO, Jepsen DB (1998) Effects of seasonality and fish movement on tropical river food webs. J Fish Biol 53(Supplement A):267-296

Yoshioka H (2016) Mathematical analysis and validation of an exactly solvable model for upstream migration of fish schools in one-dimensional rivers. Math Biosci 281:139-148

Yoshioka H, Shirai T (2015) On analytical viscosity solution to a 1-D Hamilton-Jacobi-Bellman equation for upstream migration of individual fishes in rivers. In: Proceedings of EMAC2015, p 52

Yoshioka H, Unami K, Fujihara M (2015) Mathematical and numerical analyses on a Hamilton-Jacobi-Bellman equation governing ascending behaviour of fishes. RIMS Kôkyûroku 1946:250-260

Yoshioka H, Yaegashi Y, Unami K, Fujihara M (2016a) Identifying the cost function for upstream migration of individual fishes in 1-D open channels based on an optimal control theory. J JSCE Ser B1 72:1147-1152

Yoshioka H, Yaegashi Y, Unami K, Fujihara M (2016b) Application of stochastic control theory to biophysics of fish migration around a weir equipped with fishways. In: Zhang L (ed) Theory, methodology, tools and applications for modeling and simulation of complex systems: communications in computer and information science, vol 645. Springer Science+Business Media, Singapore, pp 190-200

$\mathrm{Yu} \mathrm{B}, \mathrm{Xu} \mathrm{L}$ (2016) Review of ecological compensation in hydropower development. Renew Sustain Energy Rev 55:729-738 